\title{
OGLE-2013-BLG-0102LA,B: MICROLENSING BINARY WITH COMPONENTS AT STAR/BROWN DWARF AND BROWN DWARF/PLANET BOUNDARIES
}

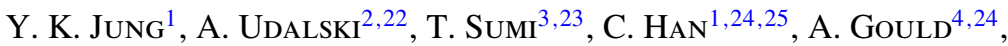 \\ AND \\ J. Skowron ${ }^{2}$, S. KozŁowski ${ }^{2}$, R. Poleski ${ }^{2,4}$, Ł. WyrzyKowski ${ }^{2,5}$, M. K. Szymański ${ }^{2}$, G. Pietrzyński ${ }^{2,6}$, \\ I. Soszyński ${ }^{2}$, K. UlaczyK ${ }^{2}$, P. Pietrukowicz ${ }^{2}$, P. Mróz ${ }^{2}$, M. KubiaK $^{2}$ \\ (The OGLE COLlaboration) \\ F. Abe ${ }^{7}$, D. P. Bennett ${ }^{8}$, I. A. Bond ${ }^{9}$, C. S. Botzler ${ }^{10}$, M. Freeman ${ }^{10}$, A. Fukui ${ }^{11}$, D. Fukunaga ${ }^{7}$, Y. Itow ${ }^{7}$, N. Koshimoto ${ }^{3}$, \\ P. Larsen ${ }^{5}$, C. H. Ling ${ }^{9}$, K. Masuda ${ }^{7}$, Y. Matsubara ${ }^{7}$, Y. Muraki ${ }^{7}$, S. Namba ${ }^{3}$, K. Ohnishi ${ }^{12}$, L. Philpott $^{13}$, \\ N. J. Rattenbury ${ }^{10}$, To. Saito ${ }^{14}$, D. J. Sullivan ${ }^{15}$, D. Suzuki ${ }^{3}$, P. J. Tristram ${ }^{16}$, N. Tsurumi ${ }^{7}$, \\ K. WAdA ${ }^{3}$, N. YAMAI ${ }^{17}$, P. C. M. Yock ${ }^{10}$, A. Yonehara ${ }^{17}$ \\ (The MOA COLlaboration) \\ M. Albrow ${ }^{18}$, J.-Y. Choi ${ }^{1}$, D. L. DePoy ${ }^{19}$, B. S. GAudi ${ }^{4}$, K.-H. Hwang ${ }^{1}$, C.-U. LeE ${ }^{20}$, H. PARK ${ }^{1}$, \\ S. OWEn ${ }^{4}$, R. W. PogGE ${ }^{4}$, I.-G. SHIN ${ }^{1}$, AND J. C. YeE Yet $^{41}$ \\ (THE $\mu$ FUN COLlaboration) \\ ${ }^{1}$ Department of Physics, Chungbuk National University, Cheongju 371-763, Korea; cheongho@ astroph.chungbuk.ac.kr \\ 2 Warsaw University Observatory, Al. Ujazdowskie 4, 00-478 Warszawa, Poland \\ ${ }^{3}$ Department of Earth and Space Science, Osaka University, Osaka 560-0043, Japan \\ ${ }^{4}$ Department of Astronomy, Ohio State University, 140 West 18th Avenue, Columbus, OH 43210, USA \\ ${ }^{5}$ Institute of Astronomy, University of Cambridge, Madingley Road, Cambridge CB3 OHA, UK \\ ${ }^{6}$ Departamento de Astronomia, Universidad de Concepción, Casilla 160-C, Concepción, Chile \\ ${ }^{7}$ Solar-Terrestrial Environment Laboratory, Nagoya University, Nagoya 464-8601, Japan \\ ${ }^{8}$ Department of Physics, University of Notre Dame, 225 Nieuwland Science Hall, Notre Dame, IN 46556-5670, USA \\ ${ }^{9}$ Institute of Information and Mathematical Sciences, Massey University, Private Bag 102-904, North Shore Mail Centre, Auckland, New Zealand \\ ${ }^{10}$ Department of Physics, University of Auckland, Private Bag 92-019, Auckland 1001, New Zealand \\ ${ }^{11}$ Okayama Astrophysical Observatory, National Astronomical Observatory of Japan, Asakuchi, Okayama 719-0232, Japan \\ 12 Nagano National College of Technology, Nagano 381-8550, Japan \\ ${ }^{13}$ Department of Earth, Ocean, and Atmospheric Sciences, University of British Columbia, 2207 Main Hall, Vancouver, BC V6T 1Z4, Canada \\ ${ }_{14}$ Tokyo Metropolitan College of Aeronautics, Tokyo 116-8523, Japan \\ ${ }^{15}$ School of Chemical and Physical Sciences, Victoria University, Wellington, New Zealand \\ ${ }^{16}$ Mt. John University Observatory, P.O. Box 56, Lake Tekapo 8770, New Zealand \\ ${ }^{17}$ Department of Physics, Faculty of Science, Kyoto Sangyo University, Kyoto 603-8555, Japan \\ ${ }^{18}$ Department of Physics and Astronomy, University of Canterbury, Private Bag 4800, Christchurch, New Zealand \\ ${ }^{19}$ Department of Physics and Astronomy, Texas A\&M University, College Station, TX 77843-4242, USA \\ ${ }^{20}$ Korea Astronomy and Space Science Institute, 776 Daedukdae-ro, Daejeon, Korea \\ ${ }^{21}$ Harvard-Smithsonian Center for Astrophysics, 60 Garden Street, Cambridge, MA 02138, USA \\ Received 2014 July 29; accepted 2014 November 4; published 2015 January 8
}

\begin{abstract}
We present an analysis of the gravitational microlensing event OGLE-2013-BLG-0102. The light curve of the event is characterized by a strong short-term anomaly superposed on a smoothly varying lensing curve with a moderate magnification $A_{\max } \sim 1.5$. It is found that the event was produced by a binary lens with a mass ratio between the components of $q=0.13$ and the anomaly was caused by the passage of the source trajectory over a caustic located away from the barycenter of the binary. Based on the analysis of the effects on the light curve due to the finite size of the source and the parallactic motion of the Earth, we determine the physical parameters of the lens system. The measured masses of the lens components are $M_{1}=0.096 \pm 0.013 M_{\odot}$ and $M_{2}=0.012 \pm 0.002 M_{\odot}$, which correspond to near the hydrogen-burning and deuterium-burning mass limits, respectively. The distance to the lens is $3.04 \pm 0.31 \mathrm{kpc}$ and the projected separation between the lens components is $0.80 \pm 0.08 \mathrm{AU}$.
\end{abstract}

Key words: binaries: general - brown dwarfs - gravitational lensing: micro

\section{INTRODUCTION}

It is generally agreed that stars form through the collapse of gas clouds in the interstellar medium while planets form either through coagulation of dust in protostellar disks or gravitational instabilities of the gas disk. By contrast, there exist diverse mechanisms proposed to explain the formation

\footnotetext{
${ }^{22}$ Optical Gravitational Lensing Experiment (OGLE) Collaboration.

${ }^{23}$ Microlensing Observations in Astrophysics (MOA) Collaboration.

${ }^{24}$ Microlensing Follow Up Network ( $\mu$ FUN) Collaboration.

${ }^{25}$ Corresponding author.
}

of objects with masses between stars and planets, i.e., very low-mass (VLM) stars and brown dwarfs, including dynamical interaction (e.g., Boss 2001; Reipurth \& Clarke 2001; Umbreit et al. 2005; Bate 2009), photoionizing radiation (e.g., Hester et al. 1996; Whitworth \& Zinnecker 2004), disk instability (e.g., Goodwin \& Whitworth 2007; Stamatellos et al. 2007), turbulent fragmentation (e.g., Padoan \& Nordlund 2004; Hennebelle \& Chabrier 2008), etc.; see Luhman (2012) for detailed review.

In order to test the formation theories of VLM objects, various types of observations are needed. In this sense, observational studies of binaries composed of VLM objects are important 
because predictions of binary properties vary considerably among formation theories. For example, the dynamical interaction and disk instability mechanisms predict few widely separated binaries while other mechanisms make no concrete predictions concerning such a trend. Furthermore, with the exception of extreme microlensing events (Gould 1997; Gould et al. 2009) and astrometric microlensing (Cushing et al. 2014), binaries provide the only channel to measure model-independent physical parameters including masses.

Unfortunately, comprehensive studies of VLM binaries have been difficult due to the lack of unbiased samples. Most known low-mass binaries have been discovered through direct imaging (e.g., Close et al. 2003, 2007). Due to their intrinsic nature, faint or dark VLM objects cannot be seen by this method and thus the sample favors binaries with luminous components and roughly equal masses, although some binaries found by other methods, such as the "astrometric variable" method (e.g., Dahn et al. 2008; Dupuy \& Liu 2012; Sahlmann et al. 2013) and "blended light spectroscopy" (e.g., Burgasser et al. 2010; Bardalez Gagliuffi et al. 2014), have little or no such bias. In addition, since it is difficult to detect closely separated ( $\lesssim 1 \mathrm{AU})$ binary systems due to the limitation set by angular resolution, the sample is biased toward widely separated binaries. For the same reason, the sample is confined to binaries in the solar neighborhood. Furthermore, spectroscopic radial-velocity observations for these objects are difficult due to their faintness, and thus it is difficult to precisely measure their masses.

Because of the difference in sensitivity from other methods, gravitational microlensing provides a complementary tool to study VLM objects. Microlensing occurs due to the bending of light caused by the gravity of a lensing object located between an observer and a lensed star (source). As a result, the phenomenon does not depend on the brightness of lensing objects, making it possible to detect faint and even dark objects. Lensing events occur on a Galactic scale, and thus the method can be used to detect VLM binaries distributed over a wide range of Galactocentric distances. In addition, the method is sensitive to tight binaries with small separations (Choi et al. 2013). Furthermore, for well-observed binary-lens events, it is possible to precisely measure binary masses without additional follow-up observations. This method has already demonstrated the usefulness in detecting VLM objects existing in various forms, e.g., a free-floating brown dwarf (Gould et al. 2009), binary brown dwarfs (Choi et al. 2013), brown dwarfs around stars (Shin et al. 2012; Street et al. 2013), and a brown dwarf orbited by a planetary mass object (Han et al. 2013).

In this paper, we report a low-mass binary discovered from the observation of the microlensing event OGLE-2013-BLG-0102. We demonstrate that the binary is composed of a primary near the hydrogen-burning limit and a companion near the deuteriumburning limit.

\section{OBSERVATION}

The lensing event OGLE-2013-BLG-0102 occurred on a star located toward the Galactic bulge direction with the equatorial coordinates $(\alpha, \delta)_{\mathrm{J} 2000}=\left(17^{\mathrm{h}} 52^{\mathrm{m}} 07^{\mathrm{s}} .08,-31^{\circ} 41^{\prime} 26^{\prime \prime} .1\right)$, corresponding to the Galactic coordinates $(l, b)=(358.36,-2.626)$. The event was first discovered by the Optical Gravitational Lensing Experiment (OGLE: Udalski 2003) collaboration from the survey conducted toward the Galactic bulge field using the $1.3 \mathrm{~m}$ Warsaw Telescope at Las Campanas Observatory in Chile; the discovery was announced to the microlensing community on 2013 March 2. The event was independently discovered by the
Microlensing Observations in Astrophysics (MOA: Bond et al. 2001; Sumi et al. 2003) collaboration using the $1.8 \mathrm{~m}$ Telescope at Mt. John Observatory in New Zealand and dubbed MOA-2013-BLG-127. The MOA group noted that the event had undergone an anomaly and issued a further alert for followup observations. No immediate follow-up observation could be performed because the anomaly occurred during the very early bulge season when the duration of the bulge visibility was short and telescopes for follow-up observations were not fully operational. Fortunately, the cadence of the survey observations was high enough to delineate the anomaly covering both the rising and falling parts of the anomaly.

Based on modeling of the light curve conducted by the end of the anomaly, it was suggested that the anomaly was produced by the crossing of a caustic ${ }^{26}$ formed by a binary lens where the mass ratio between the components is low. In response to the potential importance of the event, the Microlensing Follow-Up Network (Gould et al. 2006) collaboration took multiband images (14 images in the $I$ band and 12 images in the $V$ band) during the period from April 25 to June 29 using the $1.3 \mathrm{~m}$ SMARTS telescope of Cerro Tololo InterAmerican Observatory in Chile to obtain the color information of the source star. The event lasted more than 100 days after the anomaly. From multiple-stage real-time modeling of the light curve conducted as the event progressed, it was suggested that higher-order effects would be needed to precisely describe the event.

Figure 1 shows the light curve of the event. It is characterized by a strong short-term anomaly centered at $\mathrm{HJD}^{\prime}=\mathrm{HJD}-$ $2450000 \sim 6374.5$ superposed on a smooth brightness variation of the source star. The event lasted throughout the whole bulge season and the anomaly lasted $\sim 4$ days.

Data sets used for analysis were reduced using photometry codes developed by the individual groups, which are based on difference image analysis (Alard \& Lupton 1998). For the use of data sets processed by different photometry systems, we readjust error bars. In this process, we first add a quadratic term so that the cumulative distribution of $\chi^{2}$ sorted by magnification becomes approximately linear. We then rescale the error bars so that $\chi^{2}$ per degree of freedom $\left(\chi^{2} /\right.$ dof $)$ for each data set becomes unity (Dong et al. 2007). The first process is needed to ensure that the dispersion of data points is consistent with error bars regardless of the source brightness. The second process is required to ensure that each data set is fairly weighted according to error bars. We eliminate $3 \sigma$ outliers in the analysis in order to minimize their effect on modeling.

\section{ANALYSIS}

Under the approximation that the relative lens-source motion is rectilinear, the light curve of a binary-lens event is described by seven standard parameters; see the Appendix for graphical presentation of the parameters. In our modeling of the light curve, we use the center of mass of the binary lens as the reference position.

We search for the set of lensing parameters that best describes the observed light curve over multiple stages. In the first stage, we explore the $\chi^{2}$ surface in the parameter space and locate all of the possible local minima by conducting a grid search for a subset of the lensing parameters. At the second stage, we further refine each local minimum. At the last stage,

\footnotetext{
26 The caustic represents the closed curve of formally infinite magnification
} on the source plane. 


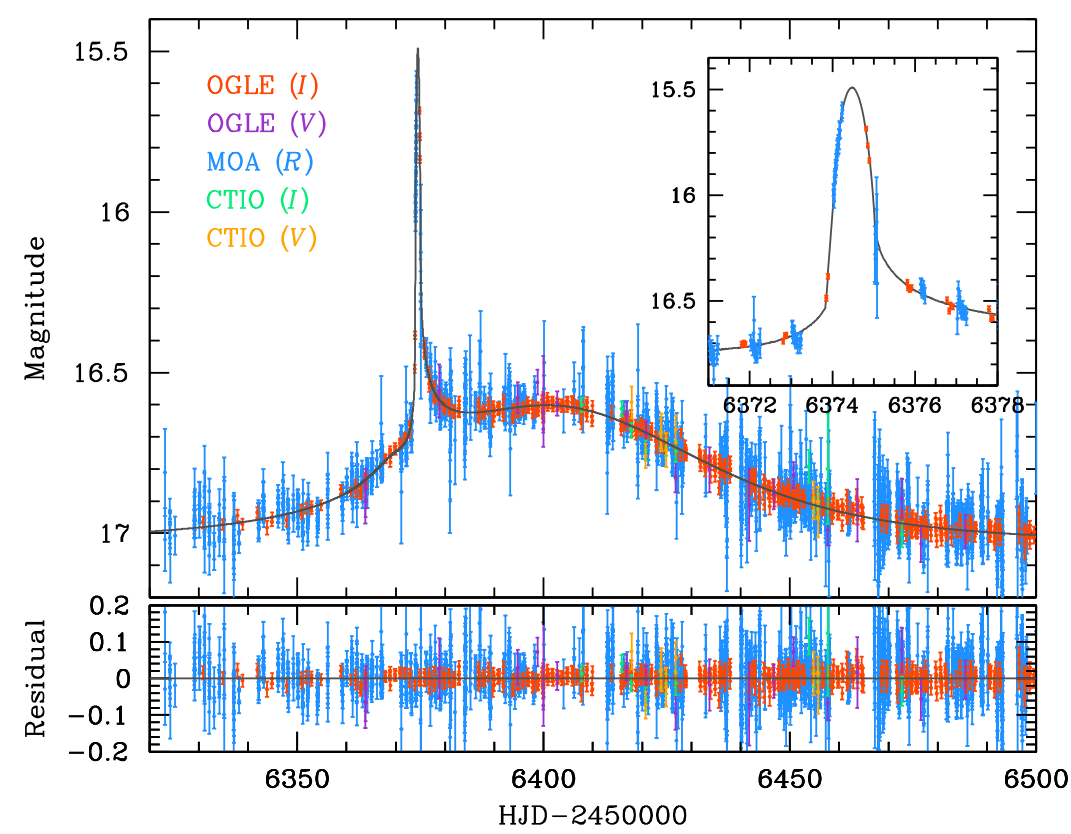

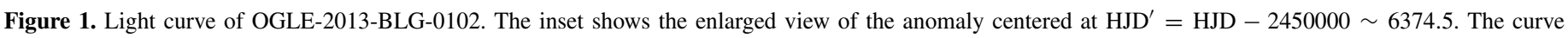
superposed on the data is the best-fit model.

we identify the global minimum by comparing $\chi^{2}$ values of the individual local solutions. The grid search of the first stage is performed in the parameter space $(s, q, \alpha)$ for which the lensing magnifications can vary dramatically with small changes to the parameters. For the other parameters, for which lensing magnifications vary smoothly for the changes of the parameters, we search for solutions by minimizing $\chi^{2}$ using a Markov Chain Monte Carlo (MCMC). Once a solution is found, the uncertainty of each lensing parameter is estimated based on the distribution of parameters derived from the corresponding MCMC chain.

To model the anomaly, which was produced by the caustic crossing of the source star, we need to consider finite-source effects. To compute finite magnifications, we use the numerical inverse ray-shooting method (Kayser et al. 1986; Schneider $\&$ Weiss 1987) for the central region of the perturbation and the semi-analytic hexadecapole approximation (Pejcha \& Heyrovský 2009; Gould 2008) for the vicinity of the perturbation. We account for the surface-brightness variation of the source caused by limb darkening by modeling the surface-brightness profile using a linear limb-darkening law. We adopt the limb-darkening coefficients $\left(u_{V}, u_{R}, u_{I}\right)=$ $(0.81,0.73,0.63)$ from Claret $(2000)$ using $T_{\text {eff }}=4500 \mathrm{~K}$ and $\log g=2$, where $T_{\text {eff }}$ and $\log g$ are derived from the dereddened brightness and color of the source star (see Section 4). For the MOA data taken using a non-standard filter system, we use $u_{R I}=\left(u_{R}+u_{I}\right) / 2=0.68$.

For some binary-lensing events, the seven basic parameters are not adequate to precisely describe lensing light curves. These cases often occur for long timescale events where the assumption of rectilinear lens-source motion is no longer valid. Parallax effects occur due to the change of the observer's position caused by the orbital motion of the Earth around the Sun (Gould 1992; Alcock et al. 1995), leading to non-rectilinear source motion. Similarly, lens orbital effects also cause non-rectilinear source motion due to the change of the lens positions caused by the orbital motion of the lens (Dominik 1998; Albrow et al. 2000; Shin et al. 2011; Jung et al. 2013; Park et al. 2013). Parallax effects are described by two parameters $\pi_{\mathrm{E}, N}$ and $\pi_{\mathrm{E}, E}$, which are the two components of the lens parallax vector $\boldsymbol{\pi}_{\mathrm{E}}$ projected onto the sky along the north and east equatorial coordinates, respectively. The magnitude of $\boldsymbol{\pi}_{\mathrm{E}}$ corresponds to the ratio of the lens-source relative parallax $\pi_{\text {rel }}$ to the angular Einstein radius $\theta_{\mathrm{E}}$, i.e.,

$$
\pi_{\mathrm{E}}=\frac{\pi_{\mathrm{rel}}}{\theta_{\mathrm{E}}}, \quad \pi_{\mathrm{rel}}=\mathrm{AU}\left(\frac{1}{D_{\mathrm{L}}}-\frac{1}{D_{\mathrm{S}}}\right),
$$

where $D_{\mathrm{L}}$ and $D_{\mathrm{S}}$ are the distances to the lens and source, respectively (Gould 2004). The direction of $\boldsymbol{\pi}_{\mathrm{E}}$ corresponds to the lens-source relative motion. To first-order approximation, the lens-orbital effects are described by two parameters $d s / d t$ and $d \alpha / d t$ that represent the change rates of the projected binary separation and the source-trajectory angle, respectively.

Measurements of higher-order effects are important for the determination of the physical lens parameters. By measuring the normalized source radius $\rho_{*}$ from the analysis of the light curve affected by finite-source effects, one can measure the Einstein radius by

$$
\theta_{\mathrm{E}}=\frac{\theta_{*}}{\rho_{*}},
$$

where the angular source radius $\theta_{*}$ is derived from the information about the source star (see Section 4). Then, along with the lens parallax measured from the analysis of the long-term deviation caused by parallax effects, the mass and distance to the lens are determined by

$$
M_{\mathrm{tot}}=\frac{\theta_{\mathrm{E}}}{\kappa \pi_{\mathrm{E}}}, \quad D_{\mathrm{L}}=\frac{\mathrm{AU}}{\pi_{\mathrm{E}} \theta_{\mathrm{E}}+\pi_{\mathrm{S}}},
$$

respectively. Here, $\kappa=4 G /\left(c^{2} \mathrm{AU}\right)$ and $\pi_{\mathrm{S}}=\mathrm{AU} / D_{\mathrm{S}}$ is the parallax of the source star.

We test various models considering individual and combinations of higher-order effects. In the "standard" model, the light curve is fitted based on the seven standard lensing parameters. In the "parallax" and "orbit" models, we separately consider the parallax and lens-orbital effects. Finally, 
Table 1

Lensing Parameters

\begin{tabular}{|c|c|c|c|c|c|c|c|}
\hline \multirow[t]{2}{*}{ Parameters } & \multirow[t]{2}{*}{ Standard } & \multicolumn{2}{|c|}{ Parallax } & \multicolumn{2}{|c|}{ Orbit } & \multicolumn{2}{|c|}{ Orbit+Parallax } \\
\hline & & $u_{0}>0$ & $u_{0}<0$ & $u_{0}>0$ & $u_{0}<0$ & $u_{0}>0$ & $u_{0}<0$ \\
\hline$\overline{\chi^{2} / \mathrm{dof}}$ & $13472.0 / 13396$ & $13428.8 / 13394$ & $13433.9 / 13394$ & $13459.8 / 13394$ & $13458.6 / 13394$ & $13405.0 / 13392$ & $13422.0 / 13392$ \\
\hline$t_{0}\left(\mathrm{HJD}^{\prime}\right)$ & $6406.71 \pm 0.17$ & $6406.47 \pm 0.19$ & $6406.38 \pm 0.16$ & $6405.54 \pm 0.18$ & $6405.51 \pm 0.18$ & $6406.18 \pm 0.20$ & $6406.30 \pm 0.17$ \\
\hline$u_{0}$ & $0.809 \pm 0.010$ & $0.832 \pm 0.011$ & $-0.827 \pm 0.006$ & $0.871 \pm 0.008$ & $-0.875 \pm 0.009$ & $0.811 \pm 0.011$ & $-0.812 \pm 0.008$ \\
\hline$t_{\mathrm{E}}$ (days) & $38.5 \pm 0.3$ & $38.9 \pm 0.4$ & $39.3 \pm 0.3$ & $38.3 \pm 0.3$ & $38.2 \pm 0.3$ & $37.6 \pm 0.4$ & $37.1 \pm 0.4$ \\
\hline$s$ & $0.594 \pm 0.003$ & $0.595 \pm 0.003$ & $0.596 \pm 0.002$ & $0.575 \pm 0.002$ & $0.573 \pm 0.003$ & $0.607 \pm 0.004$ & $0.607 \pm 0.002$ \\
\hline$q$ & $0.146 \pm 0.005$ & $0.112 \pm 0.005$ & $0.109 \pm 0.003$ & $0.072 \pm 0.002$ & $0.067 \pm 0.002$ & $0.130 \pm 0.007$ & $0.143 \pm 0.002$ \\
\hline$\alpha(\mathrm{rad})$ & $6.36 \pm 0.02$ & $6.24 \pm 0.02$ & $-6.22 \pm 0.01$ & $6.09 \pm 0.01$ & $-6.07 \pm 0.01$ & $6.36 \pm 0.02$ & $-6.39 \pm 0.01$ \\
\hline$\rho_{*}\left(10^{-3}\right)$ & $10.28 \pm 0.21$ & $10.27 \pm 0.30$ & $9.91 \pm 0.21$ & $8.16 \pm 0.24$ & $7.85 \pm 0.16$ & $11.12 \pm 0.24$ & $11.71 \pm 0.33$ \\
\hline$\pi_{\mathrm{E}, N}$ & $\ldots$ & $0.11 \pm 0.07$ & $-0.01 \pm 0.05$ & $\ldots$ & $\ldots$ & $0.48 \pm 0.05$ & $-0.44 \pm 0.07$ \\
\hline$\pi_{\mathrm{E}, E}$ & $\ldots$ & $-0.17 \pm 0.03$ & $-0.18 \pm 0.03$ & $\ldots$ & $\ldots$ & $-0.06 \pm 0.03$ & $-0.20 \pm 0.03$ \\
\hline$d s / d t\left(\mathrm{yr}^{-1}\right)$ & $\ldots$ & $\ldots$ & $\ldots$ & $0.44 \pm 0.05$ & $0.51 \pm 0.03$ & $-0.31 \pm 0.05$ & $-0.48 \pm 0.06$ \\
\hline$d \alpha / d t\left(\mathrm{yr}^{-1}\right)$ & $\ldots$ & $\cdots$ & $\ldots$ & $-1.48 \pm 0.07$ & $1.64 \pm 0.04$ & $-0.46 \pm 0.18$ & $0.05 \pm 0.13$ \\
\hline
\end{tabular}

Note. HJD' $=$ HJD -2450000 .

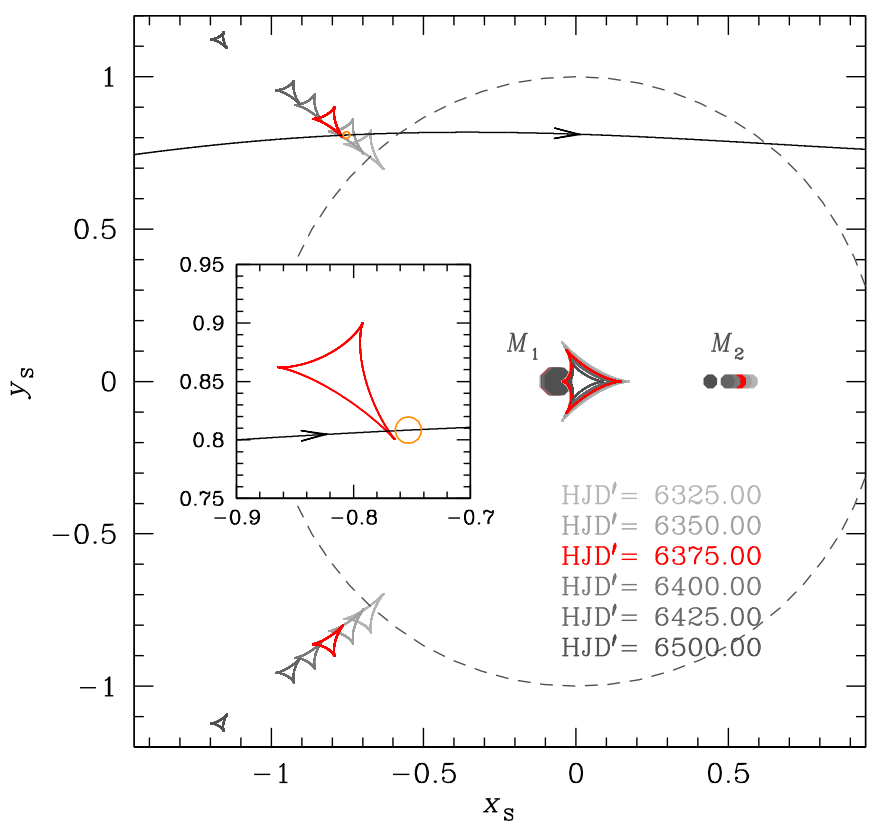

Figure 2. Geometry of the source trajectory (curve with an arrow) with respect to the lens components $\left(M_{1}\right.$ and $\left.M_{2}\right)$ and caustics (closed figures composed of concave curves). The dashed circle represents the Einstein ring. The coordinates are centered at the barycenter of the binary lens and all lengths are scaled to the Einstein radius corresponding to the total mass of the binary. Caustics vary in time due to the lens orbital motion. The red caustics correspond to the time of the anomaly. The inset shows the enlarged view of the source star's caustic crossing. The empty orange circle represents the source size relative to the caustic.

in the "orbit+parallax" model, we consider both the parallax and orbital effects. When the higher-order effects are considered, we test two solutions resulting from "ecliptic degeneracy" (Skowron et al. 2011). The two solutions resulting from this degeneracy have almost identical parameters, except $\left(u_{0}, \alpha, \pi_{\mathrm{E}, N}, d \alpha / d t\right) \rightarrow-\left(u_{0}, \alpha, \pi_{\mathrm{E}, N}, d \alpha / d t\right)$.

\section{PHYSICAL PARAMETERS}

In Table 1, we list the results of analysis for the models that we tested. The model light curve of the best-fit solution (orbit+parallax with $u_{0}>0$ ) is superposed on the data in Figure 1. Figure 2 shows the geometry of the lens system where the source trajectory with respect to the lens positions and caustics are presented. It is found that the event was produced by a binary with a mass ratio between the components is $q=0.13$. The projected separation between the binary components is $s=0.61$, which is less than the Einstein radius. In this case, the caustic is composed of three closed curves where one is located near the barycenter of the binary lens and the other two are located away from the central region. The anomaly was produced by the passage of the source trajectory over one of the outer caustics.

We find that the higher-order effects improve the fit. As measured by the $\chi^{2}$ difference from the standard model, the fit improvements are $\Delta \chi^{2}=43.2$ and 13.4 when the parallax and orbital effects are separately considered. When both effects are simultaneously considered, the improvement is $\Delta \chi^{2}=67.0$, which is $>8 \sigma$. While this is formally significant, careful diagnosis of the signal is needed because in microlensing subtle systematic trends might masquerade as signals. We therefore check the possibility of systematics by inspecting where the signal of the higher-order effects comes from. If systematics in the data affected the fit, then the signal would come from localized epochs of the event. By contrast, if the signal is due to genuine higher-order effects, then it would come throughout the event because both the orbital motions of the Earth and the lens have long-term effects on the lensing light curve. In Figure 3, we present the cumulative distribution of $\Delta \chi^{2}$ as a function of time. Although there exist several local fluctuations, and thus the possibility of systematics cannot be completely ruled out, $\chi^{2}$ improvement occurs throughout the event, suggesting that the signals of higher-order effects are real. Since the anomaly is well covered, finite-source effects are clearly detected, yielding a normalized source radius of $\rho_{*}=(11.1 \pm 0.2) \times 10^{-3}$.

With both the finite-source and parallax effects measured, the mass and distance to the lens are estimated using the relations in Equations (3). For this, we estimate the angular source radius $\theta_{*}$ based on the color and brightness. The angular Einstein radius is estimated following two steps. In the first step, we estimate the dereddened color $(V-I)_{0}$ and the brightness $I_{0}$ of the source star by using the centroid of bulge clump giants for which its dereddened color, $(V-I)_{0, \mathrm{c}}=$ 1.06 , and brightness, $I_{0, \mathrm{c}}=14.45$, are known from independent measurements (Bensby et al. 2013; Nataf et al. 2013). This method is valid because the source and bulge giants are at nearly same distances, and thus experience almost the same extinction (Yoo et al. 2004). The estimated color and brightness of the source star are $(V-I, I)_{0}=(1.08,14.97)$. Figure 4 


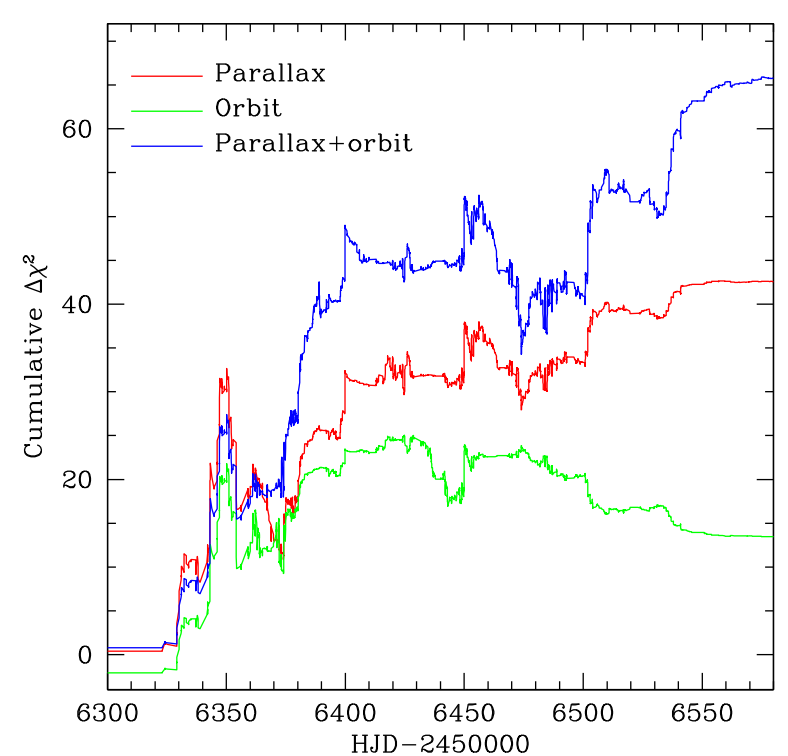

Figure 3. Cumulative distributions of $\Delta \chi^{2}$ between the models considering higher-order effects relative to the standard model as a function of time.

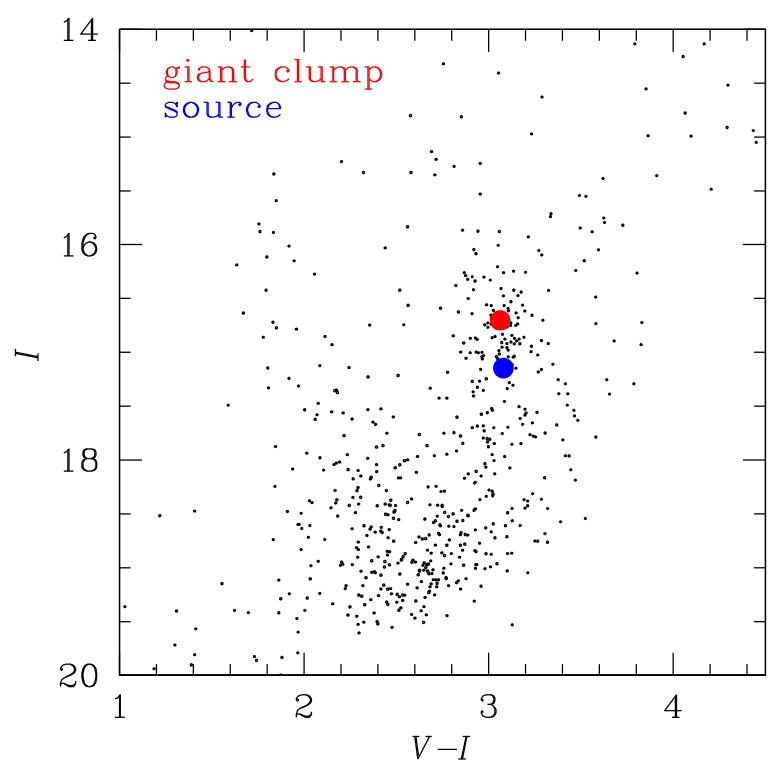

Figure 4. Instrumental color-magnitude diagram of stars in the region around the lensed star. The locations of the lensed star (source) and the centroid of the giant clump are marked.

shows the location of the source star in the color magnitude of neighboring stars with respect to the centroid of clump giants, indicating that the source is a K-type giant. In the second step, we convert $V-I$ into $V-K$ using the color-color relation (Bessell \& Brett 1988). Then the angular source radius is estimated by adopting the relation between $V-K$ and $\theta_{*}$ provided by Kervella et al. (2004). The derived angular source radius is $\theta_{*}=4.80 \pm 0.41 \mu \mathrm{as}$. The error on $\theta_{*}$ comes from three major sources: (1) the uncertainty in the source flux $f_{\mathrm{S}},(2)$ the uncertainty involved with the conversion from color to $\theta_{*}$, and (3) other auxiliary uncertainties concerned with processes such as positioning the centroid of giant stars, $V-I$ to $V-K$ conversion, etc. We estimate that the uncertainty in $f_{\mathrm{S}}$ is $\sigma_{f_{\mathrm{S}}}=5 \%$. Uncertainties of the microlensing colors $\sigma_{(V-I)_{0}}=0.07$ mag (Bensby et al. 2013) and brightness $\sigma_{I_{0, \mathrm{c}}}=0.09 \mathrm{mag}$ (Nataf et al. 2013) of clump giant stars contribute $\sim 6 \%$ error in $\theta_{*}$ measurement.
Table 2

Physical Parameters

\begin{tabular}{lcc}
\hline \hline Parameters & $u_{0}>0$ & $u_{0}<0$ \\
\hline Angular Einstein radius (mas) & $0.43 \pm 0.04$ & $0.41 \pm 0.03$ \\
Geocentric proper motion $\left(\mathrm{mas} \mathrm{yr}^{-1}\right)$ & $4.19 \pm 0.37$ & $4.06 \pm 0.34$ \\
Heliocentric proper motion $\left(\mathrm{mas} \mathrm{yr}^{-1}\right)$ & $4.30 \pm 0.38$ & $3.69 \pm 0.31$ \\
Total mass $\left(M_{\odot}\right)$ & $0.108 \pm 0.014$ & $0.104 \pm 0.017$ \\
Primary mass $\left(M_{\odot}\right)$ & $0.096 \pm 0.013$ & $0.091 \pm 0.015$ \\
Companion mass $\left(M_{\odot}\right)$ & $0.012 \pm 0.002$ & $0.013 \pm 0.002$ \\
Distance $(\mathrm{kpc})$ & $3.04 \pm 0.31$ & $3.15 \pm 0.37$ \\
Projected separation $(\mathrm{AU})$ & $0.80 \pm 0.08$ & $0.79 \pm 0.09$ \\
$(\mathrm{KE} / \mathrm{PE})_{\perp}$ & $0.028 \pm 0.006$ & $0.037 \pm 0.005$ \\
\end{tabular}

By considering other factors, we adopt the uncertainty from factors (2) and (3) as 7\%. The estimated source radius corresponds to the angular Einstein radius $\theta_{\mathrm{E}}=0.43 \pm 0.04$ mas.

In Table 2, we summarize the determined physical quantities of the lens system. We present two sets of quantities corresponding to the $u_{0}>0$ and $u_{0}<0$ solutions resulting from the ecliptic degeneracy. It is found that the $u_{0}>0$ model is preferred by $\Delta \chi^{2}=17.0$. This is formally at the $>4 \sigma$ level, but one cannot completely rule out the $u_{0}<0$ model considering possible systematics in data. However, we note that both solutions result in similar physical quantities. According to the best-fit model, the masses of the lens components are $M_{1}=0.096 \pm 0.013 M_{\odot}$ and $M_{2}=0.012 \pm 0.002 M_{\odot}$. The distance to the lens is $D_{\mathrm{L}}=3.04 \pm 0.31 \mathrm{kpc}$ and the projected separation between the lens components is $r_{\perp}=s D_{\mathrm{L}} \theta_{\mathrm{E}}=0.80 \pm$ $0.08 \mathrm{AU}$. In order to check the validity of the solution, we also present the projected kinetic to potential energy ratio, which is computed by

$$
\left(\frac{\mathrm{KE}}{\mathrm{PE}}\right)_{\perp}=\frac{\left(r_{\perp} / \mathrm{AU}\right)^{2}}{8 \pi^{2}\left(M_{\mathrm{tot}} / M_{\odot}\right)}\left[\left(\frac{1}{s} \frac{d s}{d t}\right)^{2}+\left(\frac{d \alpha}{d t}\right)^{2}\right],
$$

where $M_{\text {tot }}$ is the total mass of the binary lens (Dong et al. 2009). We note that the lensing parameters $d s / d t$ and $d \alpha / d t$ are determined from modeling considering the orbital motion of the lens. The ratio should be less than unity to be a bound system. The measured ratio is $(\mathrm{KE} / \mathrm{PE})_{\perp}<1.0$ and thus meets the condition of boundness. Its small value tends to imply that the true separation is several times larger than the projected separation.

In Figure 5, we compare the physical parameters of OGLE2013-BLG-0102L to those of low-mass binaries from the VLM binaries archive ${ }^{27}$ and other references (Basri \& Martín 1999; Lane et al. 2001; Burgasser et al. 2008, 2012; Faherty et al. 2011). Also marked are the three low-mass microlensing binaries: OGLE-2009-BLG-151L and OGLE-2011-BLG-420L reported by Choi et al. (2013) and OGLE-2012-BLG-0358L reported by Han et al. (2013). It is found that the microlensing binaries are located in the low-mass, close-separation, and low-mass-ratio regions in the parameter space. Among known VLM binaries, we find that only "Cha $\mathrm{H} \alpha$ 8" has similar physical parameters: $r_{\perp}=1.3 \mathrm{AU}, M_{1}=0.1 M_{\odot}, M_{2}=0.019 M_{\odot}$ (Joergens 2006; Joergens \& Müller 2007). However, this binary was discovered in a star-forming cloud, and thus is very young. Therefore, the reported VLM binary demonstrates that microlensing provides an important method that can complement other methods.

\footnotetext{
27 http://www.vlmbinaries.org
} 

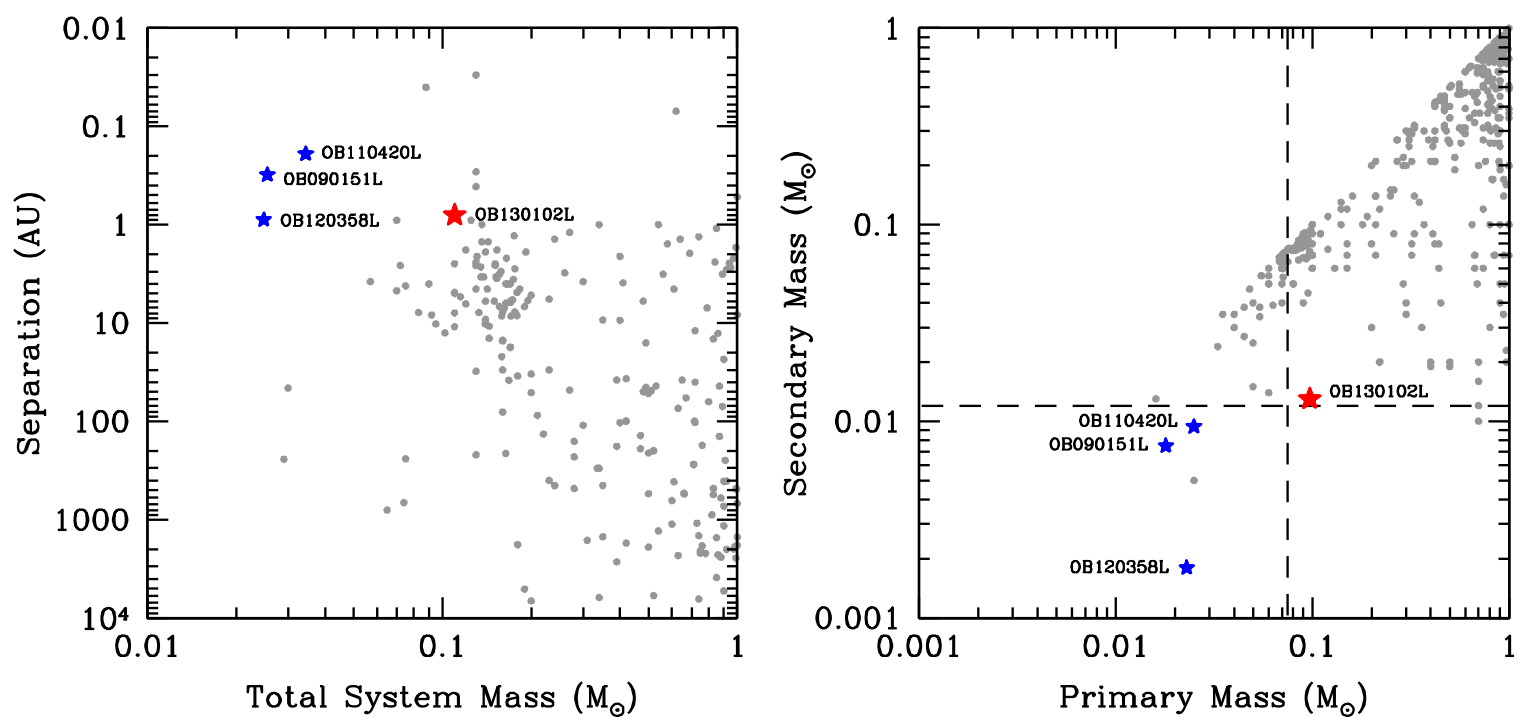

Figure 5. Total mass vs. separation (left panel) and primary vs. secondary masses (right panel) for a compilation of low-mass binaries. Microlensing binaries are denoted in "star" marks while those discovered by other methods are marked by dots. The red star is the microlensing binary reported in this work and the three blue stars are the binaries reported by Choi et al. (2013) and Han et al. (2013). The vertical and horizontal dashed lines represent the star/brown dwarf and brown dwarf/planet boundaries, respectively.

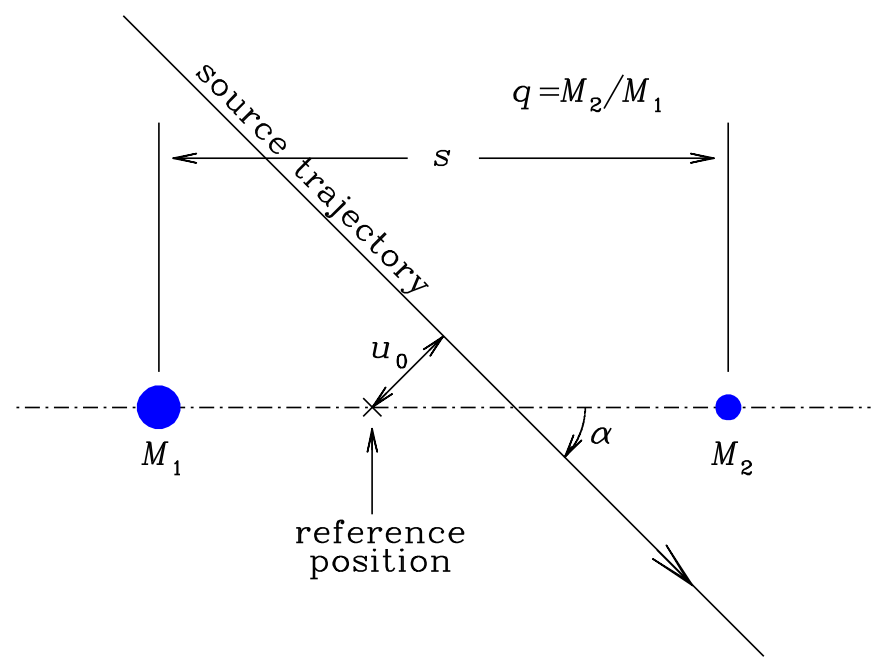

Figure 6. Graphical presentation of binary-lensing parameters. The filled dots marked by $M_{1}$ and $M_{2}$ represent the locations of the lens components. The straight line with an arrow is the source trajectory.

The discovered binary is of scientific interest because the masses of the primary and the companion correspond to the upper and lower limits of brown dwarfs, respectively. Although there exist some dispute, the popular convention for the division between low-mass stars and brown dwarfs is $\sim 0.075 M_{\odot}$, below which hydrogen fusion reaction in cores does not occur (Burrows et al. 1997), while the convention for the division between brown dwarfs and giant planets is $\sim 0.012 M_{\odot}\left(13 M_{\mathrm{J}}\right)$, below which deuterium burning cannot be ignited (Spiegel et al. 2011). Then, the individual binary components of OGLE2013-BLG-0102L have masses near the hydrogen-burning and deuterium-burning mass limits, respectively.

\section{CONCLUSION}

We found a VLM binary from the observation and analysis of the microlensing event OGLE-2013-BLG-0102. The event was characterized by a strong short-term anomaly superposed on a smoothly varying lensing curve with a moderate magnification. It was found that the event was produced by a binary object with a mass ratio between the components of $q=0.13$ and the anomaly was caused by the passage of the source trajectory over a caustic located away from the barycenter of the binary lens. By measuring deviations in the lensing light curve caused by both finite-source and parallax effects, we determined the physical parameters of the lens. It was found that the lens is composed of objects with masses $M_{1}=0.096 \pm 0.013 M_{\odot}$ and $M_{2}=0.012 \pm 0.002 M_{\odot}$, which correspond to the hydrogenburning and deuterium-burning limits, respectively. The binary is located at a distance of $3.04 \pm 0.31 \mathrm{kpc}$ and the projected separation between the components is $0.80 \pm 0.08 \mathrm{AU}$.

Work by C.H. was supported by Creative Research Initiative Program (2009-0081561) of National Research Foundation of Korea. A.G. and B.S.G. acknowledge support from NSF AST-1103471. B.S.G., A.G., and R.W.P. acknowledge support from NASA grant NNX12AB99G. The OGLE project has received funding from the European Research Council under the European Community's Seventh Framework Programme (FP7/2007-2013)/ERC grant agreement No. 246678 to A.U. The MOA experiment was supported by grants JSPS22403003 and JSPS23340064. T.S. acknowledges the support of JSPS 24253004. T.S. is supported by the grant JSPS23340044. Y.M. acknowledges support from JSPS grants JSPS23540339 and JSPS19340058. Work by J.C.Y. was performed in part under contract with the California Institute of Technology (Caltech) funded by NASA through the Sagan Fellowship Program.

\section{APPENDIX}

\section{BINARY-LENSING PARAMETERS}

For the basic description of binary-lens events, seven lensing parameters are needed. Three of these parameters describe the source-lens approach: the time of the closest source approach to a reference position of the lens, $t_{0}$, the separation between the 
source and the reference position at $t_{0}, u_{0}$ (impact parameter), and the timescale for the source to cross the Einstein radius corresponding to the total mass of the binary lens, $t_{\mathrm{E}}$ (Einstein timescale). Another three parameters describe the binary lens: the mass ratio, $q=M_{2} / M_{1}$, the projected separation between the lens components, $s$, and the angle between the source trajectory and the binary axis, $\alpha$ (source trajectory angle); see the graphical presentation of the parameters in Figure 6. We note that parameters $u_{0}$ and $s$ are normalized to the angular Einstein radius $\theta_{\mathrm{E}}$. The last parameter is the source radius normalized to the Einstein radius, $\rho_{*}$ (normalized source radius).

\section{REFERENCES}

Albrow, M. D., Beaulieu, J.-P., Caldwell, J. A. R., et al. 2000, ApJ, 534, 894 Alcock, C., Allsman, R. A., Alves, D., et al. 1995, ApJL, 454, L125

Alard, C., \& Lupton, R. H. 1998, ApJ, 503, 325

Bardalez Gagliuffi, D. C., Burgasser, A. J., Gelino, C. R., et al. 2014, ApJ, 794, 143

Basri, G., \& Martín, E. L. 1999, AJ, 118, 2460

Bate, M. R. 2009, MNRAS, 392, 590

Bensby, T., Yee, J. C., Feltzing, S., et al. 2013, A\&A, 549, 147

Bessell, M. S., \& Brett, J. M. 1988, PASP, 100, 1134

Bond, I. A., Abe, F., Dodd, R. J., et al. 2001, MNRAS, 327, 868

Boss, A. P. 2001, ApJL, 551, L167

Burgasser, A. J., Cruz, K. L., Cushing, M., et al. 2010, ApJ, 710, 1142

Burgasser, A. J., Liu, M. C., Ireland, M. J., et al. 2008, ApJ, 681, 579

Burgasser, A. J., Luk, C., Dhital, S., et al. 2012, ApJ, 757, 110

Burrows, A., Marley, M., Hubbard, W. B., et al. 1997, ApJ, 491, 856

Choi, J.-Y., Han, C., Udalski, A., et al. 2013, ApJ, 768, 129

Claret, A. 2000, A\&A, 363, 1081

Close, L. M., Siegler, N., Freed, M., et al. 2003, ApJ, 587, 407

Close, L. M., Zuckerman, B., Song, I., et al. 2007, ApJ, 660, 1492

Cushing, M. C., Kirkpatrick, J. D., Gelino, C. R., et al. 2014, AJ, 147, 113

Dahn, C. C., Harris, H. C., Levine, S. E., et al. 2008, ApJ, 686, 548

Dominik, M. 1998, A\&A, 329, 361
Dong, S., Gould, A., Udalski, A., et al. 2009, ApJ, 695, 970 Dong, S., Udalski, A., Gould, A., et al. 2007, ApJ, 664, 862 Dupuy, T. J., \& Liu, M. C. 2012, ApJS, 201, 19

Faherty, J. K., Burgasser, A. J., Bochanski, J. J., et al. 2011, A\&A, 141, 71

Goodwin, S. P., \& Whitworth, A. 2007, A\&A, 466, 943

Gould, A. 1992, ApJ, 392, 442

Gould, A. 1997, ApJ, 480, 188

Gould, A. 2004, ApJ, 606, 319

Gould, A. 2008, ApJ, 681, 1593

Gould, A., Udalski, A., An, D., et al. 2006, ApJ, 644, 37

Gould, A., Udalski, A., Monard, B., et al. 2009, ApJL, 698, L147

Han, C., Jung, Y. K., Udalski, A., et al. 2013, ApJ, 778, 38

Hennebelle, P., \& Chabrier, G. 2008, ApJ, 684, 395

Hester, J. J., Scowen, P. A., Sankrit, R., et al. 1996, AJ, 111, 2349

Joergens, V. 2006, A\&A, 446, 1165

Joergens, V., \& Müller, A. 2007, ApJ, 666, 113

Jung, Y. K., Han, C., Gould, A., et al. 2013, ApJL, 768, L7

Kayser, R., Refsdal, S., \& Stabell, R. 1986, A\&A, 166, 36

Kervella, P., Bersier, D., Mourard, D., et al. 2004, A\&A, 428, 587

Lane, B. F., Zapatero Osorio, M. R., Britton, M. C., et al. 2001, ApJ, 560, 390

Luhman, K. L. 2012, ARA\&A, 50, 65

Nataf, D. M., Gould, A., Fouqué, P., et al. 2013, ApJ, 769, 88

Padoan, P., \& Nordlund, A. 2004, ApJ, 617, 559

Park, H., Udalski, A., Han, C., et al. 2013, ApJ, 778, 134

Pejcha, O., \& Heyrovský, D. 2009, ApJ, 690, 1772

Reipurth, B., \& Clarke, C. 2001, AJ, 122, 432

Sahlmann, J., Lazorenko, P. F., Ségransan, D., et al. 2013, A\&A, 556, 133

Schneider, P., \& Weiss, A. 1987, A\&A, 171, 49

Shin, I.-G., Han, C., Gould, A., et al. 2012, ApJ, 760, 116

Shin, I.-G., Udalski, A., Han, C., et al. 2011, ApJ, 735, 85

Skowron, J., Udalski, A., Gould, A., et al. 2011, ApJ, 738, 87

Spiegel, D. S., Burrows, A., \& Milsom, J. A. 2011, ApJ, 727, 57

Stamatellos, D., Hubber, D. A., \& Whitworth, A. P. 2007, MNRAS, 382, L30

Street, R., Choi, J.-Y., Tsapras, Y., et al. 2013, ApJ, 763, 67

Sumi, T., Abe, F., Bond, I. A., et al. 2003, ApJ, 591, 204

Udalski, A. 2003, AcA, 53, 291

Umbreit, S., Burkert, A., Henning, T., et al. 2005, ApJ, 623, 940

Whitworth, A. P., \& Zinnecker, H. 2004, A\&A, 427, 299

Yoo, J., DePoy, D. L., Gal-Yam, A., et al. 2004, ApJ, 603, 139 